\title{
A Distributed Mapping Control of Identifiers and Locators for Future Mobile Internet
}

\author{
Nak-Jung Choi*, Heeyoung Jung**, Seok-Joo Koh* \\ * School of Computer Science and Engineering, Kyungpook National University, Korea \\ ** Electronics and Telecommunications Research Institute, Korea \\ peaceful7007@gmail.com, hyjung@etri.re.kr, sjkoh@knu.ac.kr
}

\begin{abstract}
The future Internet will be evolved to mobile-oriented environments, and thus the mobility support is a key issue in the design of future Internet. This paper proposes a distributed identifier-locator mapping system (DMS) for the future mobileoriented Internet environment, which is designed on the identifier-locator (ID-LOC) separation principle. The proposed DMS scheme is implemented over Linux platform by using the OpenFlow and Click Router software modules. From experimentations over the real testbed network of Korea Research Education Network (KOREN), we can see that the proposed DMS scheme can effectively perform the mobility management operations for mobile Internet hosts. For performance comparison, we can see that the proposed DMS scheme reduces the signaling delays for mobility management, including ID-LOC mapping update/query and data delivery, compared with the existing mobility control schemes, Proxy Mobile IP (PMIP).
\end{abstract}

Keywords - ID-LOC Separation, Distributed Mapping System, OpenFlow, Experimentation

\section{INTRODUCTION}

It is noted that the current Internet was historically designed for fixed network environment, rather than for the mobile network environment. This has enforced Inter-net to add a lot extensional features to satisfy the mobility requirements, as shown in the example of Mobile IP (MIP) [1, 2]. However, this patch-on approach seems to be just a temporal heuristic to the problems in the mobile environment, rather than an optimization. Thus, a variety of research activities have been made to design the future Internet for mobile environment, which include eMobility [3], 4WARD [4], FIND [5], MobilityFirst [6], GENI [7], and AKARI [8]. It is also noted that many challenging works are in progress with the identifier-locator separation principle, as shown in Host Identity Protocol (HIP) [9], Locator-Identifier Separation Protocol (LISP) [10], and Identifier-Locator Network Protocol (ILNP) [11].

With these observations, we design the architecture of Mobile Oriented Future Internet (MOFI) [12] to support the mobile environment of future Internet. The MOFI is designed with the following functional blocks: Host Identifier and Local Locator (HILL) and Distributed Mapping System
(DMS). The details of the MOFI architecture are described in [12].

This paper specifies a distributed mapping system (DMS) to support the identifier-locator (ID-LOC) mapping management based on the ID-LOC separation. The DMS control operations include HID-LOC binding for HID-LOC registration and LOC query for data delivery. The DMS functional entities include Local Mapping Controller (LMC) at AR and Global Mapping Controller (GMC) at gateway (GW). For intra-domain communication, LMC is used to maintain the Local Map Register (LMR) to keep the list of HID-LOC mapping information for hosts. For inter-domain communication, GMC is used to maintain a Global Map Register (GMR) for HID-LOC mapping management for the hosts in the other domain.

This paper is organized as follows. Section 2 briefly summarizes the MOFI architecture. In Section 3 we describe the HID-LOC mapping control operations for DMS. Section 4 describes the Linux-based implementation using the OpenFlow and Click Router platform and the experimental results performed over the Korea Research and Education Network (KOREN) network. Section 5 concludes this paper.

\section{MOFI ARCHITECTURE OVERVIEW}

\section{A. Host ID and Local Locator (HILL)}

HID is used to identify a mobile host. LOC is used to represent the location of a host in the network and also used for delivery of data packets. Figure 1 shows the data delivery operations with global HID-based communication and local LOC-based routing in MOFI.

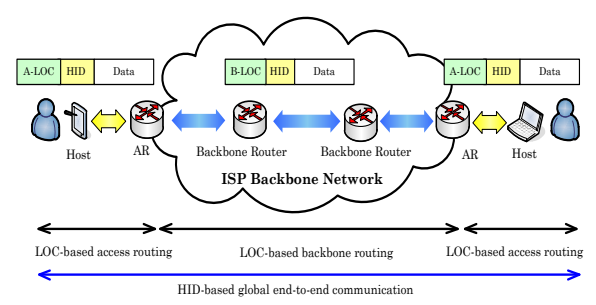

Figure 1. HID-based Communication and LOC-based Routing 
In the figure, the communication between two hosts is performed with HIDs, whereas LOCs are used for packet delivery. For packet delivery, an access LOC (A-LOC) is used as LOC within access network, whereas the IP address of AR will be used as backbone LOC (B-LOC) in the backbone network.

\section{B. Distributed HID-LOC Mapping System (DMS)}

Figure 2 shows the overall network model for HID-LOC mapping control of DMS, in which a domain represents the network domain of an ISP.

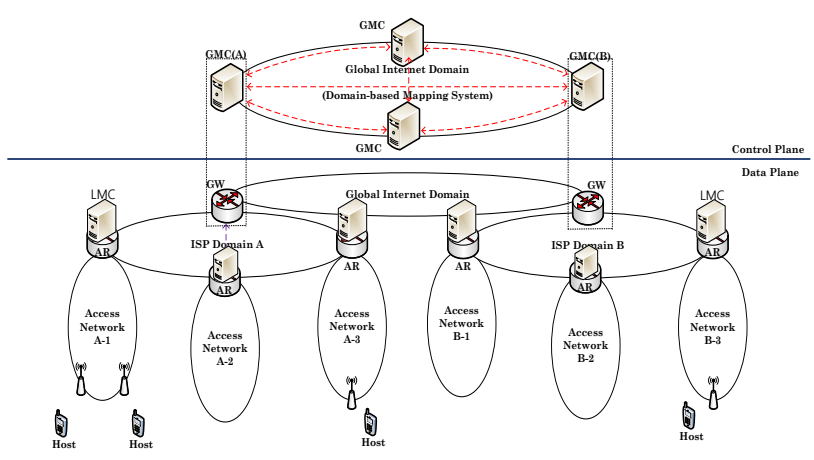

Figure 2. Mapping Control Model of DMS.

In the figure, the control plane is separated from the data plane. In the data plane, an Access Router (AR) maintains a Local Mapping Controller (LMC) that maintains the list of HID-LOC of the locally attached hosts. Each domain gateway (GW) maintains its Global Mapping Controller (GMC) for HID-LOC mapping of all hosts.

\section{Query-First Data Delivery (QFDD)}

The current MIP mobility protocol is based on the 'datadriven packet delivery' model, which induces non-optimal routes. In MIP, a mobile node (MN) updates its $\mathrm{LOC}(\mathrm{CoA})$ to HA, when it is attached to a Foreign Agent (FA). The correspondent node $(\mathrm{CN})$ sends data packets to HA, and HA will forward these packets to $\mathrm{MN}$ via $\mathrm{FA}$, which induces a non-optimal route.

In QFDD of MOFI, the LOC query operation will be performed before data transmission to find the optimal route. The HID and LOC of MN is registered with the DMS agent. When $\mathrm{CN}$ sends a data packet to $\mathrm{MN}$, the access router of $\mathrm{CN}$ will perform the LOC query operation with the DMS agents to find the LOC of MN. After that, the data packet is delivered to MN by using the optimal route.

\section{HID-LOC MAPPING CONTROL IN DMS}

The HID-LOC mapping control by DMS is further divided into 'intra-domain' and 'inter-domain' cases. This paper focuses on the 'inter-domain' mapping control. The discussion of intra-domain case is omitted in this paper. The intra-domain operations are performed based on the OpenFlow Click system. Table 1 shows the list of the messages used for mapping control.
Table 1. Control Messages for Mapping Control

\begin{tabular}{|l|l|l|l|}
\hline Message & Full Name & From & To \\
\hline HBR & HID Binding Request & Host/GMC & GMC/GMC \\
HBA & HID Binding ACK & GMC/GMC & GMC/Host \\
LQR & LOC Query Request & GMC & GMC \\
LQA & LOC Query Ack & GMC & GMC \\
\hline
\end{tabular}

The HBR and HBA control messages are exchanged between host and GMC, or between GMCs. On the other hands, the LQR and LQA messages are exchanged between GMCs. Each control message is encapsulated into UDP.

\section{A. HID-LOC Binding Operation}

With network attachment of host, the HID-LOC operation is performed, in which HID and LOC of the host will be registered with LMC. LMC will maintain and update its Local Mapping Register (LMR) the information of bindings between HIDs and LOCs for all of the hosts that are attached to the $\mathrm{LMC} / \mathrm{AR}$.

In HID-LOC binding, the HID Binding Request (HBR) and HID Binding ACK (HBA) messages are exchanged as shown in Figure 3. After the HID-LOC binding between host and LMC/AR, the LMC/AR of the host sends a HBR message to the GMC/GW. The HBR message contains the HID of the host and the LOC (IP address of LMC). Based on the received HBR, the GMC updates its Global Mapping Register (GMR) and responds with a HBA message to LMC of the host.

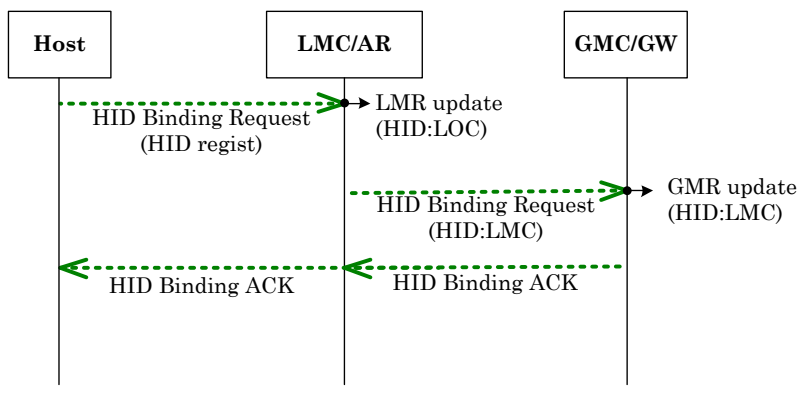

Figure 3. HID-LOC binding between host and LMC/GMC

\section{B. LOC Query and Data Delivery Operations}

In the LOC query operation, LMR and GMR will be used to find the AR and GW that a mobile host is attached to. Figure 4 shows the LOC query and data delivery operations for inter-domain case. In this case, we assume that a sending host $(\mathrm{SH})$ is attached to LMC/AR1 and a receiving host $(\mathrm{RH})$ is connected to LMC/AR2 via GW1and GW2.

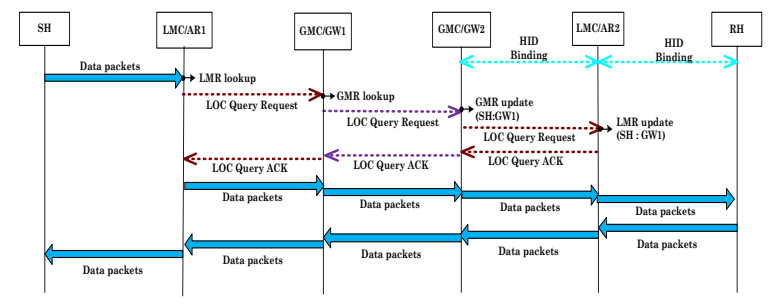

Figure 4. LOC query and data delivery for inter-domain case 
When a data packet arrives from $\mathrm{SH}$, the LMC/AR1 sends a LOC Query Request (LQR) message to GMC/GW1. On reception of a $L Q R$ message, GMC1 sends LQR to GMC2, and then GMC2 will send the LQR message to LMC/AR2. After looking up the LMR, LMC/AR2 responds with a LOC Query ACK (LQA) message to GMC/GW2. Then, GMC/GW2 sends LQA message to GMC/GW1 and further to LMC/AR1. AR1 of SH can now send a data packet to RH via GW1, GW2 and AR2.

\section{OPENFLOW-BASED IMPLEMENTATIONS}

To validate the proposed DMS scheme, the mapping control operations were implemented using UDP socket programming [13], OpenFlow [14] and Click Modular Router [15] over the Linux platform.

\section{A. Intra-domain Communications}

For intra-domain implementation, we use the OpenFlow software and an arbitrary IPv4 address as the HID. Figure 5 shows the network model for intra-domain implementation. For implementation of AR/LMC, we employ OpenFlow switch and NOX controller. The OpenFlow switch will function as AR, and it forwards the data packets. All LMRs in the domain are implemented over the OpenFlow NOX Controller. In HID-LOC binding operation, a flow table is maintained by OpenFlow Switch so as to manage all of the hosts that are attached to the AR. The LMR managed by NOX Controller will maintain the list of all hosts that are attached in the domain. The Packet-in and Packet-out messages of OpenFlow are used as the HBR and HBA messages of DMS. In the LOC query operations for data delivery, the flow table of OpenFlow switch will be updated. It is noted that the Packet-in/Flow-Mod messages of OpenFlow correspond to the LQR/LQA messages of DMS.

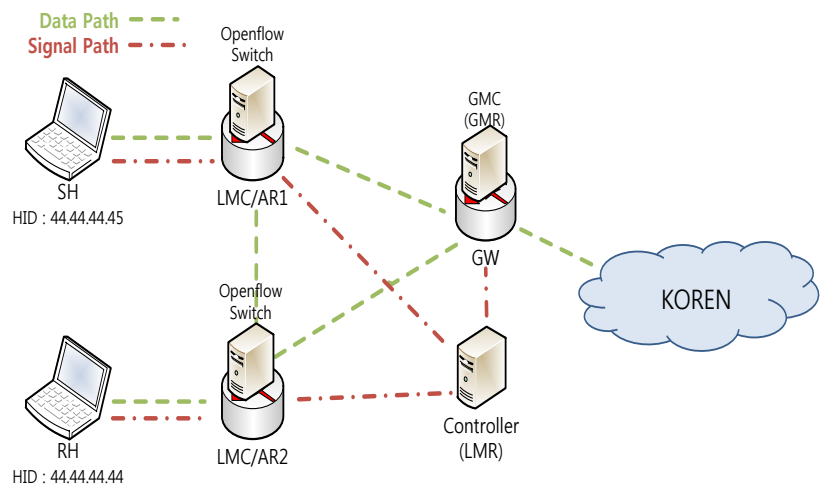

Figure 5. Intra-domain implementation by OpenFlow and Click Router

Figure 6 shows the packet capturing results for intradomain communication by using the WireShark tool [16]. The test environment is the same with Figure 5. From the figure, we can see that the data packets are delivered between the two hosts with the help of the DMS operations.

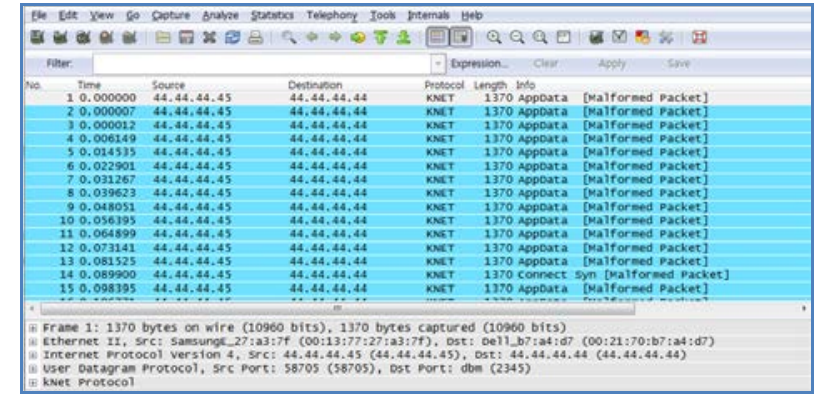

Figure 6. Intra-domain Packet Capture

Figure 7 shows the information of LMR that is updated by the HID-LOC binding operation. From the figure, 2c2c2c2c is the hexadecimal representation of the HID (44.44.44.44), and the number of 210.114.94.174 represents the location of AR that the host is attached to. From the figure, we can see that the LMR of LMC is updated as per the HID-LOC binding operation.

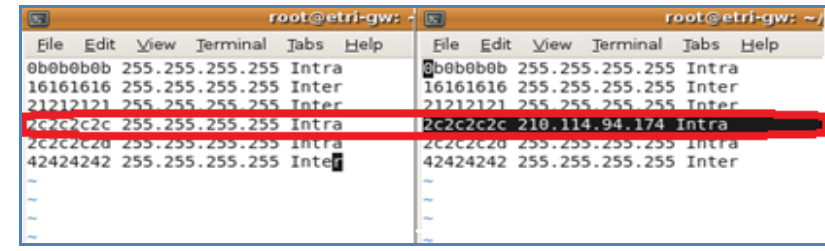

Figure 7. LMR update

\section{B. Inter-domain Communications}

For implementation of inter-domain mapping control, we use the UDP socket programming. Figure 8 shows the testbed configuration for inter-domain implementation of the proposed DMS scheme. There are the two domains, one is located at the Domain1 and another one is located at the Domain2, and the two domains are connected by Korea Research and Education Network (KOREN) [17].

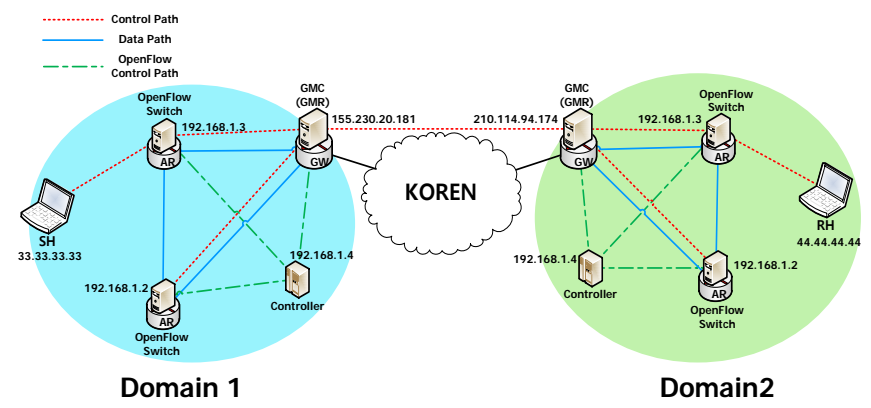

Figure 8. Inter-domain Implementation Network model

Figure 9 shows the packet capturing results that are measured by GW for inter-domain communication between Domain1 and Domain2. From the figure, we can see that the data packets are delivered between the source and destination hosts via the domain GWs. In addition, the data packets are encapsulated by using the IP-in-IP tunneling scheme at each GW. 


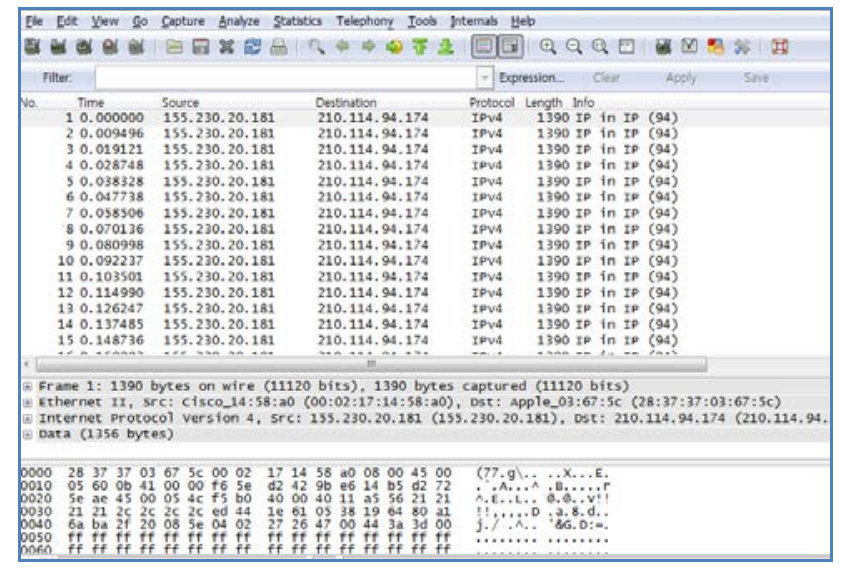

Figure 9. Inter-domain Packet Capture

Figure 10 shows the information of GMR that is updated by the HID-LOC binding operation. From the figure, 21212121 is the hexadecimal representation of the HID (33.33.33.33), and the number of 155.230.20.181 represents the location of AR that the host is attached to. From the figure, we can see that the GMR of GMC is updated as per the HID-LOC binding operation.

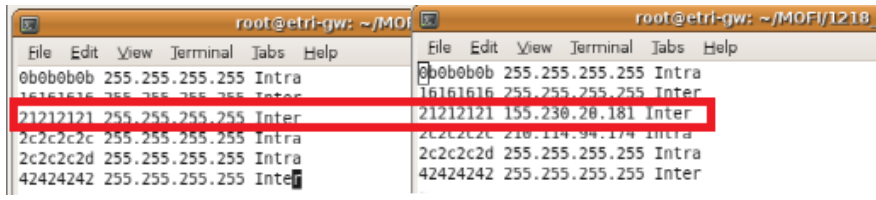

Figure 10. GMR update

\section{Experimentations of DMS Mapping Control}

Now, for experimentation of DMS mapping control at GMC, we consider the three different cases for distributed mapping control. For each test case, the GMC that is responsible for HID-LOC mapping control is selected differently, as per the modulo hash function.

In Case 1, as shown in Fig. 11, SH is attached to AR1, and $\mathrm{RH}$ is attached to AR3. It is assumed that the HID of RH is registered with GMC1, as per the hash function. Accordingly, there is no need of LOC query to $\mathrm{RH}$, when a data packet of $\mathrm{SH}$ arrives at AR1, since the HID-LOC mapping information is already maintained at GMC1. The data packets are delivered from AR1 to AR3.

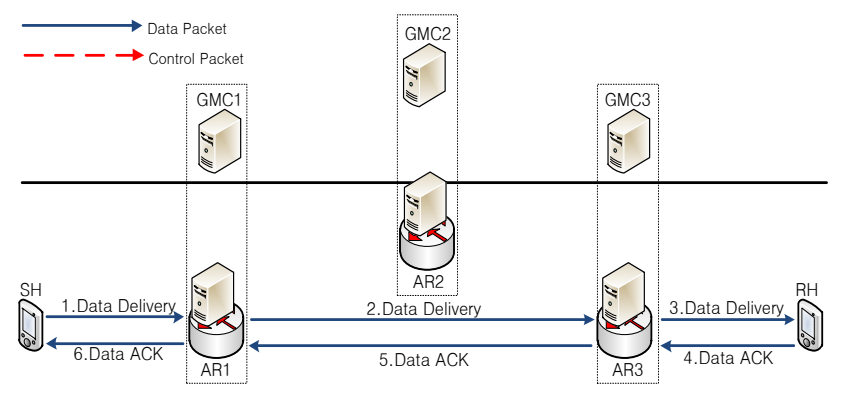

Figure 11. Case 1: No Need of LOC Query
In Case 2, as shown in Fig. 12, SH and RH are attached to AR1 and AR3, respectively. However, the HID of RH is registered with GMC2, as per the hash function. Accordingly, when a data packet of SH arrives at AR1, GMC1 will send the LQR message to GMC2. Then, GMC2 forwards this LQR message to GMC3. GMC3 responds with the LQA message to GMC1. After that, AR1 transmits the data packets to AR3.

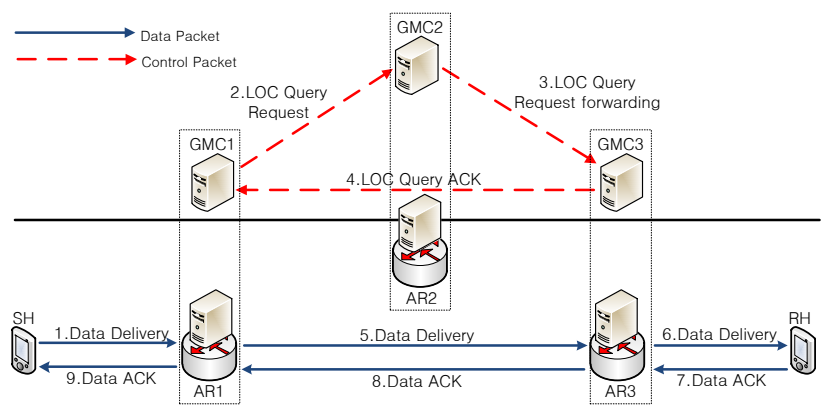

Figure 12. Case 2: LOC Query to GMC2 and to GMC3

In Case 3, as shown in Fig. 13, SH and RH are attached to AR1 and AR3, respectively. It is assumed that the HID of RH is registered with GMC3, as per the hash function. Accordingly, when a data packet of SH arrives at AR1, GMC1 will send the LQR message to GMC3. Then, GMC3 responds with the LQA message to GMC1. After that, AR1 transmits the data packets to AR3.

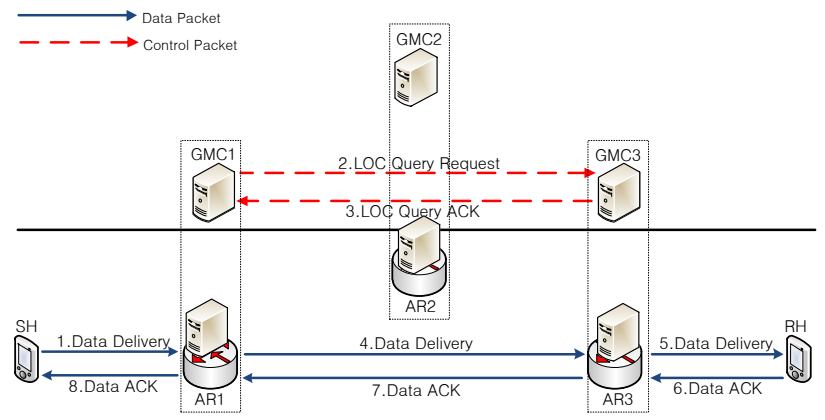

Figure 13. Case 3: LOC Query to GMC3

For comparison, we also performed the Proxy MIP (PMIP) operations, in which GMC2 plays a role of LMA and all the data packets of $\mathrm{SH}$ in AR1will be delivered to $\mathrm{RH}$ in AR3, by way of AR2. In PMIP, the LOC query operations are not performed.

In the three test cases and the PMIP case, SH transmits 10 data packets to $\mathrm{RH}$, and then we measure the packet delivery time including the LOC query operation and the data packet delivery.

Fig. 14 compares the packet delivery time for the candidate schemes, denoted by DMS: Case 1, 2, 3 and PMIP. From the figure, the PMIP gives worse performance than the three DMS schemes. This is because PMIP does not use the optimal routes, even though the LOC query operation is not required. Among the three DMS cases, the Case 1 provides the best performance. This is because the optimal route is used without using the LOC query operations in this case. In the meantime, Case 2 tends to give relatively worse performance than Case 1 
and 3, since the LOC Query message is delivered from GMC1 to GMC3 via GMC2.

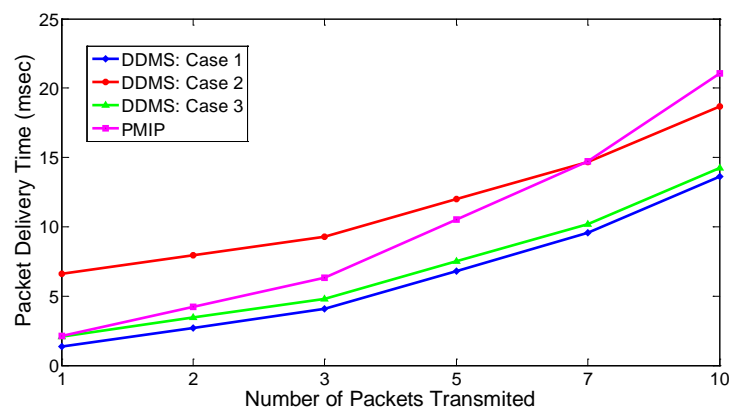

Figure 14. Packet Delivery Time for Three Test Cases and PMIP

\section{CONCLUSions}

Until now, we have described the distributed identifierlocator mapping system (DMS) for the future mobile-oriented Internet environment. For validation, we implemented the proposed DMS scheme over Linux platform by using the OpenFlow and Click Router software. From experimentations over the real testbed network of Korea Research Education Network (KOREN), we can see that the proposed DMS scheme can perform the mobility management operations effectively for mobile Internet hosts. And also we can check the MOFI DMS system is better than PMIP System.

\section{ACKNOWLEDGMENT}

This research was supported by the Basic Science Research Program of NRF (2010-0020926).

\section{REFERENCES}

[1] IETF RFC 5944, IP Mobility Support for IPv4: Revised, November 2010

[2] IETF RFC 3775, Mobility Support in IPv6, June 2004

[3] eMobility Project, http://www.emobility.eu.org/

[4] 4WARD Project, http://www.4ward-project.eu/

[5] Future Internet Design (FIND), http://www.nets-find.net/

[6] Mobility First: Future Internet Architecture, http://mobilityfirst.winlab.rutgers.edu/

[7] Global Environment for Network Innovations (GENI), http://www.geni.net/

[8] The AKARI Project, http://akari-project.nict.go.jp/eng/

[9] Host Identity Protocol (HIP), http://www.ietf.org/html.charters/hipcharter.html

[10] Locator Identifier Separation Protocol (LISP) http://www.ietf.org/html.charters/lisp-charter.html

[11] Identifier Locator Network Protocol (ILNP), http://ilnp.cs.standrews.ac.uk/

[12] Mobile Oriented Future Internet (MOFI), http://www.mofi.re.kr

[13] Behrouz A. Forouzan, TCP/IP Protocol Suit, McGraw-Hill Press, 2012.

[14] OpenFlow, http://www.openflow.org/

[15] Eddie Kohler, The Click Modular Router, Ph. D. Thesis, MIT, 2000.

[16] Wireshark, http://www.wireshark.org

[17] KOREN, http://www.koren.kr/koren/kor/

[18] ETRI Journal, v.35 n.3, Jun. 2013, Distributed Routing Based on Minimum End to End Delay for OFDMA Backhaul Mobile Mesh Networks
[19] ETRI Journal, Cost-Efficient Virtual Optical Network Embedding for Manageable Inter-Data-Center Connectivity

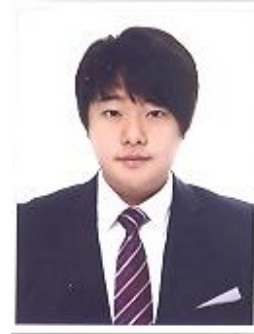

Nak Jung Choi received B.S. degree in Computer Science from Kyungpook National University in 2011. He is now a Master course in School of Electrical Engineering and Computer Science from Kyungpook National University. His current research interests include Internet Mobility, and Future Internet

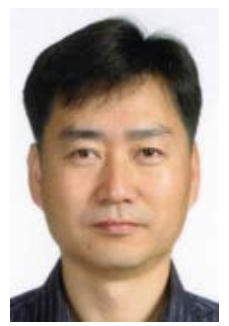

Heeyoung Jung joined Electronics and Telecommunication Research Institutes (ETRI) in 1991 after receiving his bachelor's degree from Pusan National University (PNU), and he is currently a principal research member. He received his Ph.D. degree in Information and Communications Engineering from the Chungnam National University (CNU) in 2004. His major research areas include Internet and mobile network technologies and those that are closely related to standardization activities in ITU-T, IETF, and so on. His current research topic is future Internet architecture. E-mail: hyjung@etri.re.kr.

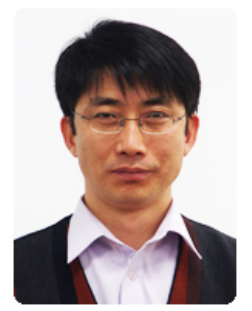

Seok Joo Koh received B.S. and M.S. degrees in Management Science from KAIST in 1992 and 1994, respectively. He also received Ph.D. degree in Industrial Engineering from KAIST in 1998. From August 1998 to February 2004, he worked for Protocol Engineering Center in ETRI. Since March 2004, he has been with the school of Electrical Engineering and Computer Science in the Kyungpook National University as an Associate Professor. His current research interests include mobility control in Future Internet, mobile SCTP, and mobile multicasting. He has also participated in the International Standardization as an editor in the ITU-T SG13 and ISO/IEC JTC1/SC6. 